\title{
Estudo da qualidade do trigo e da farinha de trigo destinada a panificação em um moinho no sul do Brasil
}

\author{
Study of the quality of wheat and flour destined for baking in a mill in the south of Brazil \\ Estudio de la calidad del trigo y la harina destinados a la elaboración de pan en un molino del sur \\ del Brasil
}

Recebido: 17/03/2021 | Revisado: 23/03/2021 | Aceito: 29/03/2021 | Publicado: 06/04/2021

\author{
Ângela Broca \\ ORCID: https://orcid.org/0000-0002-9784-241X \\ Universidade Federal Fronteira Sul, Brasil \\ E-mail: angelabroca87@yahoo.com.br \\ Larissa Canhadas Bertan \\ ORCID: https://orcid.org/0000-0003-1072-5171 \\ Universidade Federal Fronteira Sul, Brasil \\ E-mail: larissa.bertan@uffs.edu.br \\ Cátia Tavares dos Passos Francisco \\ ORCID: https://orcid.org/0000-0001-6125-2752 \\ Universidade Federal Fronteira Sul, Brasil \\ E-mail: catia.passos@uffs.edu.br
}

\begin{abstract}
Resumo
Produção e qualidade estão cada vez mais andando juntas em todos os setores da indústria alimentícia. Na produção de farinha de trigo, características tecnológicas específicas precisam ser observadas para que a produção final tenha um uso assertivo quanto a finalidade de uso. Por isso, o objetivo deste trabalho, foi avaliar a qualidade do trigo pão, beneficiado em um moinho no Sul do Brasil. Os dados foram coletados no banco de dados do moinho, das safras de 2014 a 2018, totalizando 5 anos, classificados como trigo pão. As amostras passaram por análises físico-químicas e reológicas, avaliando umidade e peso hectolitro $(\mathrm{PH})$ do grão e umidade, glúten, número de queda e alveografia da farinha. Os dados foram analisados estatisticamente por análise descritiva, análise de componentes principais (ACP), análise de agrupamento, ANOVA e teste de Tukey. A média para a umidade dos grãos encontrada foi de 12,77\%, e para o PH, a média foi de $78 \mathrm{~kg} / \mathrm{hl}$, classificando assim as amostras como Tipo 1 , conforme preconizado pela legislação vigente. A análise por PCA, identificou que glúten e número de queda, foram as variáveis menos representativas, e as variáveis da alveografia de $\mathrm{W}, \mathrm{P}, \mathrm{L}$ e $\mathrm{P} / \mathrm{L}$ foram as que mais apresentaram variabilidade, e que estas alterações não foram explicadas pelo local de origem ou safra, mesmo assim, todas ficaram dentro do recomendado, ou ideal para destinar farinha para a panificação. Demonstrando que a farinha brasileira tem qualidade e que este trabalho pode auxiliar a indústria para trabalhar mais assertivamente na produção de farinha para seus clientes finais.
\end{abstract}

Palavras-chave: PCA; Glúten; Número de queda; Variáveis; Indústria.

\begin{abstract}
Production and quality are increasingly going together in all sectors of the food industry. In the production of wheat flour, specific technological characteristics need to be observed so that the final production has an assertive use. Therefore, the objective of this work, was to evaluate the quality of the wheat bread, benefited in a mill in the South of Brazil. The data were collected in the mill's database from the 2014 to 2018 harvests, totaling 5 years, classified as bread wheat. The samples underwent physical-chemical and rheological analysis, evaluating grain moisture and $\mathrm{pH}$, gluten, number of fall and alveography of the flour. The data were statistically analyzed by descriptive analysis, principal component analysis (PCA), group analysis, ANOVA and Tukey test. The average for grain moisture was $12.77 \%$, and for $\mathrm{pH}$, the average was $78 \mathrm{~kg} / \mathrm{hl}$, thus classifying the samples as Type 1 , as recommended by current legislation. The analysis by PCA, identified that gluten and number of drops, were the least representative variables, and the variables of the alveography of $\mathrm{W}, \mathrm{P}, \mathrm{L}$ and $\mathrm{P} / \mathrm{L}$ were the most variable, and that these changes were not explained by the place of origin or crop, even so, all were within the recommended, or ideal for flour for baking. These variables may be indicative for the industry to work more assertively in the production of flour for their final customers.
\end{abstract}

Keywords: PCA; Gluten; Falling number; Variables; Industry. 


\begin{abstract}
Resumen
La producción y la calidad se mueven cada vez más juntas en todos los sectores de la industria alimentaria. En la producción de harina de trigo es necesario observar características tecnológicas específicas para que la producción final tenga un uso asertivo. Por lo tanto, el objetivo de este trabajo, era evaluar la calidad del pan de trigo, beneficiado en un molino en el sur de Brasil. Los datos se recogieron en la base de datos del molino de las cosechas de 2014 a 2018, por un total de 5 años, clasificados como trigo panificable. Las muestras se sometieron a análisis físicoquímicos y reológicos, evaluando la humedad y el pH del grano, el gluten, el número de caídas y la alveografía de la harina. Los datos fueron analizados estadísticamente por análisis descriptivo, análisis de componentes principales (ACP), análisis de grupo, ANOVA y la prueba de Tukey. La media para la humedad del grano fue de 12,77\%, y para el $\mathrm{pH}$, la media fue de $78 \mathrm{~kg} / \mathrm{hl}$, clasificando así las muestras como de Tipo 1, tal y como recomienda la legislación vigente. El análisis realizado por PCA, identificó que el gluten y el número de gotas, eran las variables menos representativas, y las variables de la alveografía de W, P, L y P/L eran las que presentaban mayor variabilidad, y que estos cambios no se explicaban por el lugar de origen o el cultivo, aún así, todas estaban dentro de lo recomendado, o ideal para destinar la harina para panificación. Estas variables pueden ser indicativas para que la industria trabaje más asertivamente en la producción de harina para sus clientes finales.
\end{abstract}

Palabras clave: PCA; Gluten; Numero decreciente; Variables; Indústria.

\title{
1. Introdução
}

O trigo (Triticum aestivum) é amplamente utilizado na alimentação, e fornece ao organismo uma gama de elementos calóricos e nutricionais para manter a ordem biológica e funcional em perfeito funcionamento. Segundo o Departamento de Agricultura dos Estados Unidos (USDA), em 2020 foram consumidos aproximadamente 750 milhões de toneladas de trigo no mundo e em torno de 12 milhões de toneladas no Brasil. A indústria moageira do trigo é a responsável pela fabricação da matéria prima mais utilizada em produtos consumidos, tanto pela população mundial, como pela população brasileira, onde o per capita de farinha de trigo ficou em torno dos 40,62 kg no território nacional em 2020 (Abitrigo, 2020; Amorin et al., 2012).

Segundo a Associação Brasileira de Trigo (ABITRIGO), até fevereiro de 2019, eram 165 moinhos em atividade em todo o Brasil, sendo que a região sul é a que compreende o maior número de moinhos (74,49\%), por ser a maior região de produção da matéria-prima. A maioria dos moinhos é de característica industrial (81\%), seguido por cooperativas (18\%) e coloniais (1\%). O grão de trigo mais usado no mercado moageiro é o Trigo Pão (62\%), seguido do Trigo melhorador (23\%), em que após o processo de moagem, transformam-se em 3 principais subprodutos: farinha, farelo e gérmen. Destes, a farinha de trigo é a mais utilizada, como matéria prima para a fabricação de pães, macarrão, biscoitos e bolos, ou outros derivados (Abitrigo 2020; Guarienti, 1996).

Como todo o produto alimentício, a farinha de trigo também é submetida a análises de qualidade, não só para garantir ao consumidor final um produto seguro, mas, também para direcionar a indústria na aplicação ideal, ou seja, para uso em massas, biscoitos ou produtos panificáveis. Segundo Caldeira et al (2000), análises físico-químicas e reológicas da farinha, determina sua destinação de uso. Neste contexto, a alveografia analisa a força da farinha, que são expressas por curvas de extensão através do aparelho alveógrafo, que indica a força do glúten. A análise do número de queda, também chamado de falling number (FN) traz como resultado a atividade da enzima $\alpha$-amilase. O percentual de glúten é determinado pela lavagem da amostra de farinha e a umidade pode ser determinada através de infravermelho ou de estufa. Após realizar essas e outras análises complementares de qualidade da farinha, como cor e cinzas, os resultados obtidos são averiguados, baseados em variáveis e critérios de cada análise, do uso específico ou das especificações próprias de cada cliente. Assim, a farinha tem a sua destinação de uso, seja para fabricação de massas, biscoitos ou panificação (Lanzarini, 2015).

Com isso, o objetivo deste trabalho foi estudar os dados obtidos sobre a qualidade do grão de trigo (potencial hectolitro e umidade) e da farinha de trigo (alveografia, Falling number e glúten), em uma indústria moageira destinada para a panificação, no sul do Brasil, provenientes do Rio Grande do Sul e Paraná, ao longo de cinco safras. 


\section{Metodologia}

Todas as amostras avaliadas foram recebidas e moídas em um moinho localizado no município de Cascavel, no Estado do Paraná, Brasil, que recebe grãos de trigo secos, de diferentes locais de produção, como do Estado do Rio Grande do Sul e do Paraná, ambos do Brasil e também, sazonalmente, de outros países, como Paraguai, Argentina e Canadá. Os dados avaliados neste trabalho foram provenientes do banco de dados do moinho, onde as análises foram realizadas entre os anos de 2014 e 2018, totalizando 5 anos. Todas as amostras foram consideradas da classe Trigo Pão, segundo a Normativa 38 (Brasil, 2010).

As amostras foram caracterizadas por análises físico-químicas e reológicas. Para isso, foi determinada a umidade do grão, pelo método de reflectância no infravermelho próximo (NIR), conforme o método 44 - 15A (AACC, 2000). Foi determinado o peso hectolitro $(\mathrm{PH})$, em balança hectolítrica com capacidade de 1/4 de litro, em cinco repetições (Santos et. al. 2015).

Para simular a moagem industrial, foi realizada a moagem experimental conforme descrito no método 26 - $10 \mathrm{~A}$ (AACC, 2000). A umidade da farinha foi determinada pela perda do peso original da amostra, utilizando-se $2 \mathrm{~g}$ de farinha, em estufa a $130^{\circ} \mathrm{C}$, durante $1 \mathrm{~h}$, conforme o método 44-15.02 (AACC, 1999).

O Índice de Queda (Falling Number - FN) foi obtido conforme método nº 56-81.03 (AACC, 1999) e do manual do equipamento, expresso em segundos (s). A determinação do teor de glúten úmido foi determinada conforme método 38-12.02 AACC (2000), por meio da lavagem de $10 \mathrm{~g}$ da farinha de trigo com solução de cloreto de sódio a $2 \%$, utilizando-se o aparelho Glutomatic Perten, seguido por centrifugação, com os resultados expressos em porcentagem (\%).

A Alveografia foi realizada no alveógrafo da marca Chopin, utilizando o método n 54-30.02 da AACC (1999), através de uma massa elaborada da pesagem de $250 \mathrm{~g}$ de farinha e o volume de solução salina $(2,5 \%)$ baseada na umidade inicial da farinha. Os parâmetros obtidos nos alveogramas foram: tenacidade (P), extensibilidade (L), expressos em milímetros e energia de deformação da massa $(\mathrm{W})$, expressa em $10^{-4} \mathrm{~J}$.

Os resultados foram analisados estatisticamente de forma descritiva e discriminativa. Para as análises descritivas foi utilizado o software Xrealstats para Excel, e a análise dos componentes principais (ACP), matriz de distâncias, ANOVA e o teste de diferença de médias, pelo teste de Tukey, usando o software PAST 4.03.

\section{Resultados e Discussão}

Com base nos resultados apresentados na Figura 1, pode-se considerar que todos os grãos de trigo investigados no presente trabalho foram considerados Tipo 1, conforme estabelecido na Instrução Normativa 38/2010 (Brasil, 2010), onde grãos acima de $78 \mathrm{~kg} / \mathrm{hL}$, são classificados como Tipo 1 . Pode-se observar que houve poucas variações, como para as amostras PR01, PR04 e RS01, sendo que para as demais, não foi possível observar os valores máximos e mínimos, devido à proximidade dos dados. O PH é utilizado pela indústria e pelo triticultor como um parâmetro de qualidade do grão do trigo, bem como a resistência a pragas e doenças e ao potencial produtivo (Guarienti, 1996). Segundo Battisti et. al. (2011), o PH é um importante parâmetro de qualidade para seleção de sementes de trigo, porém, necessita de outros parâmetros, como da dureza e do conteúdo de proteínas, para se certificar que o grão que possui PH acima de 78Kg/hl, seja selecionado para semente de uma nova safra, ou destinado à moagem para a fabricação de farinha. 
Figura 1. Peso hectolitro $(\mathrm{PH})$, apresentado pela análise Boxplot, dos grãos de trigo oriundos de diferentes locais do Estado do Paraná (PR01, PR02, PR03 e PR04) e Rio Grande do Sul (RS01 e RS02), entre os anos de 2014 e 2018, totalizando 30 análises. $\mathrm{O}$ símbolo " $\mathrm{x}$ " nas colunas representa a média.

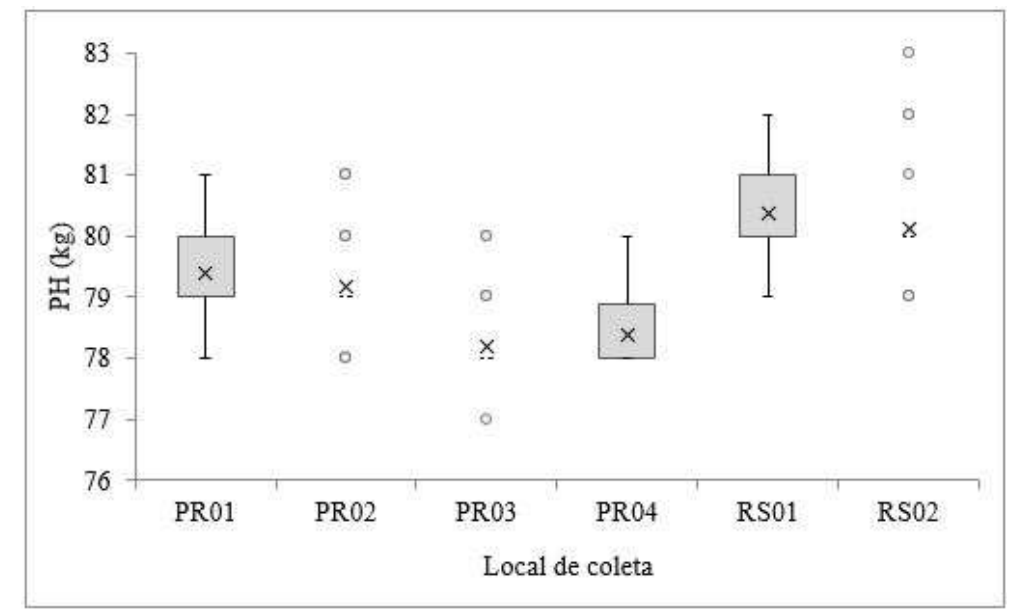

Fonte: Autores.

O grão de trigo quando colhido dentro de boas condições climáticas, apresenta umidade média entre 16 e $18 \%$ que, conforme relatado por Elias et. al. (2009), grãos obtidos dentro dessa umidade e secos por meio de secadores com condições de temperatura controlada, apresentaram melhor qualidade tecnológica e melhor conservabilidade, quando comparados a grãos colhidos com umidade de $14 \%$ e secos na própria planta. Uma umidade abaixo de $10 \%$ não é interessante, para a indústria moageira, pois aumentaria o conteúdo de água a ser usado no processo e, por consequência, o tempo de descanso para o grão, no silo, acarretando em aumento dos custos do processamento e menor extração de produto (Antunes, 2016).

Pode-se observar na Figura 2 que a umidade média dos trigos recebidos foi 12,77\%, variando de 11,60\% a 13,80\%, sendo que a maior parte das amostras analisadas apresentaram umidade entre $12 \%$ e $13 \%$. Como a umidade máxima permitida para armazenamento dos grãos no Brasil é de 13\% (Brasil, 2001), pode-se verificar que algumas amostras estavam um pouco acima deste valor, embora nenhuma tenha alcançado $14 \%$, mas que pode ser devido a técnica utilizada para a determinação de umidade, pois trata-se de uma medida indireta, onde há necessidade de comparação com método de referência e por ser uma técnica considerada de baixa sensibilidade (Jamrógiewicz, 2012). 
Figura 2. Umidade (\%), representada pela análise Boxplot, dos grãos de trigo oriundos de diferentes locais do Estado do Paraná (PR01, PR02, PR03 e PR04) e Rio Grande do Sul (RS01 e RS02), entre os anos de 2014 e 2018, totalizando 30 análises. $\mathrm{O}$ símbolo "x" nas colunas representa a média.

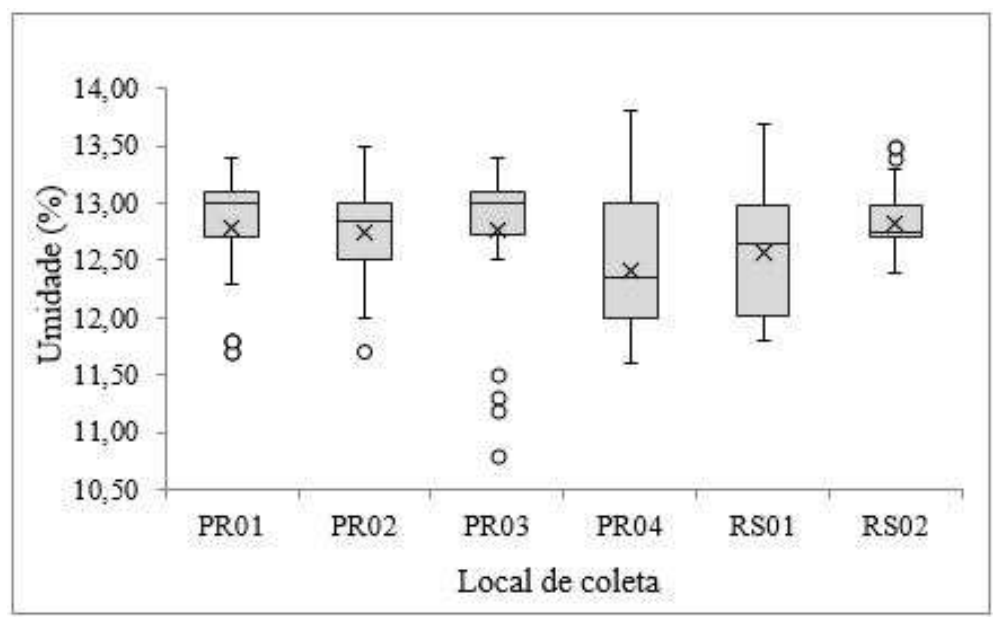

Fonte: Autores.

Como pode ser observado na Figura 3, todas as amostras de farinha de trigo foram consideradas adequadas, pois a legislação brasileira estabelece como limite máximo 15\% de umidade (Brasil, 2005), e nenhuma amostra ultrapassou este valor. No trabalho de Silva et. al. (2010), foi relatado que durante seus quatro tempos de armazenamento a umidade da farinha manteve-se entre 11,28 e 12,58\%, demonstrando a importância de um armazenamento adequado, ou seja, em local seco e ao abrigo da luz. No trabalho de Marathe et. al. (2002), verificou-se um aumento na umidade da farinha irradiada depois de três meses de armazenamento, o que segundo os autores, foi causada pela permeabilidade da embalagem na qual a farinha de trigo foi acondicionada. Quando a farinha sai do processo fabril, ela está sujeita ao ambiente onde ficará armazenada até chegar ao seu uso final, demonstrando que o ambiente de armazenamento, o tipo de embalagem, assim como todo o processo, precisa de atenção para que a farinha tenha uma vida de útil adequada.

A umidade da farinha também é fator importante quando se trata das condições microbiológicas. Hemery et. al. (2020), relataram que, dependendo das condições ambientais onde a farinha será armazenada, a embalagem a ser escolhida precisa ser levada em consideração, a fim de evitar condições favoráveis de proliferação de micro-organismos contaminantes, como Bacillus cereus, Staphylococcus aureus e Salmonella sp. Logo, estabelecer e respeitar os limites de umidade é de grande importância no armazenamento do trigo e também da farinha de trigo e bem como para futuras transações comerciais (Embrapa Trigo, 2009).

Cabe ressaltar que a moagem do grão de trigo praticamente não tem impacto sobre os aspectos microbiológicos da farinha, portanto, a qualidade do grão de trigo terá maior influência na qualidade final e na segurança dos produtos finais (Sabillon; Bianquini, 2016). 
Figura 3. Umidade representada pela análise Boxplot, da farinha de trigo oriundos de diferentes locais do Estado do Paraná (PR01, PR02, PR03 e PR04) e Rio Grande do Sul (RS01 e RS02), entre os anos de 2014 e 2018, totalizando 30 análises. O símbolo "x" nas colunas representa a média.

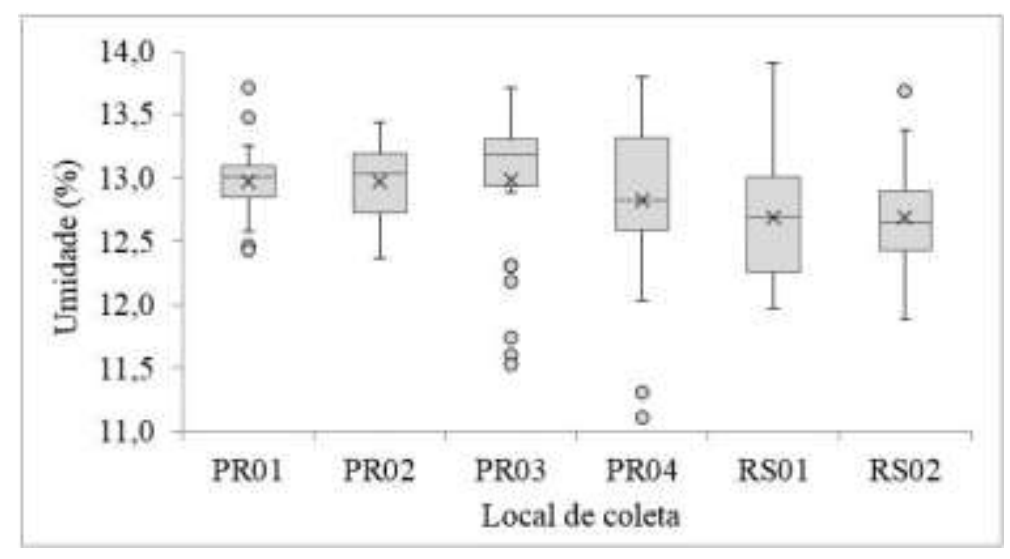

Fonte: Autores.

Foram analisados os dados padronizados, dos diferentes locais estudados, uma vez que se tratavam de variáveis diferentes com diferenças de unidades, entre as safras de 2014 e 2018 para as variáveis: Glúten, W (força), P (tenacidade), L (extensibilidade), P/L (relação entre as variáveis) e FN (Falling number) pela análise de agrupamentos (Vicini, 2005), conforme apresentado na Figura 4. A análise mostrou que os dados apresentaram uma distribuição aleatória, logo, não se pode afirmar que a variabilidade das amostras se deu por influência do clima, ou das características do local da produção, como tipo de solo, temperatura, precipitação, entre outros fatores que poderiam afetar a qualidade do trigo, pois não foi verificado nenhum grupamento que explicasse tal hipótese. Fano (2015), em seu estudo, obteve resultados inconclusivos na aplicação de enxofre, no cultivo, para a qualidade industrial do trigo, devido as condições climáticas (precipitação pluviométrica e geadas) que ocorreram durante o experimento. Boschini (2010), demonstrou que a aplicação de diferentes doses de nitrogênio e de lâminas de água na cultura do trigo, influenciou significativamente em algumas das variáveis que estava analisando, peso hectolitro (PH), energia na deformação da massa $(\mathrm{W})$ e no número de queda (FN). Logo, esses autores abordam que a qualidade do trigo é influenciada tanto pelo clima, quanto pelo tipo de cultivo, demonstrando a dificuldade de se encontrar uma tendência nos resultados. 
Figura 4. Dendograma da matriz de distâncias (distância Euclidiana), pelo método de agrupamento por ligação simples. No dendograma a escala vertical indica o nível de similaridade, e no eixo horizontal são marcados os indivíduos, na ordem em que são agrupados. As linhas verticais partem dos indivíduos, e têm altura correspondente ao nível em que os indivíduos são considerados semelhantes.
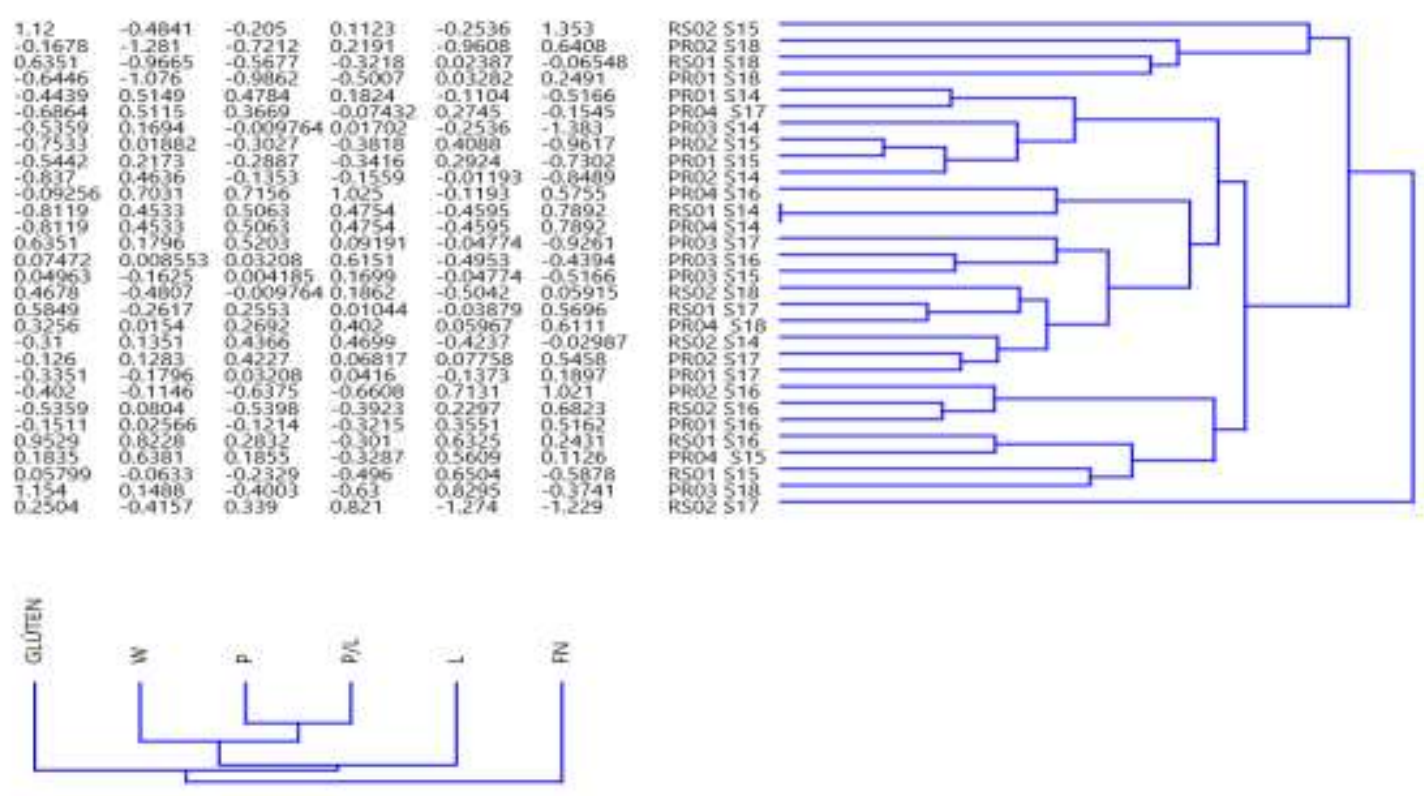

Fonte: Autores.

O Coeficiente de correlação da análise de matriz de distâncias foi de 0,8865, com intervalo de confiança de 95\%. Para as variáveis analisadas se percebeu uma similaridade entre a tenacidade $(\mathrm{P})$ e a relação $\mathrm{P} / \mathrm{L}$, estas se relacionaram com a força (W), sendo que este grupamento estava relacionado com a extensibilidade (L) e todos apresentaram relação com o glúten. Embora todas as variáveis avaliadas apresentassem algum tipo de conexão, o FN (número de queda) foi o que mais se distanciou das demais variáveis.

A fim de determinar se havia diferença entre as variáveis estudadas, realizou-se teste de diferença de médias, pelo teste de Tukey, levando-se em consideração as 30 análises realizadas para cada ponto de coleta, ao longo dos 5 anos. Como pode ser observado na Tabela 1, as variáveis W, P, L e P/L não apresentaram diferença estatística, a 95\% de confiança, mostrando que as variáveis significativas, para esta análise, foram o teor de glúten e o FN. Para o FN as amostras PR01 $(285,53 \pm 21,56)$ e PR02 $(289,4 \pm 27,31)$ apresentaram médias significativamente iguais entre si $(\mathrm{p}>0,05)$. A PR03 $(266,73 \pm 26,63)$ apresentou média significativamente menor e diferente $(\mathrm{p}<0,05)$ da amostra PR02. As amostras PR04 $(298,03 \pm 19,61)$, RS01 $(291,47 \pm 27,61)$ e RS02 $(291,87 \pm 34,60)$ foram consideradas estatisticamente iguais (p>0,05), mas apresentou diferença estatística $(\mathrm{p}<0,05)$ da amostra PR03, devido a apresentarem médias significativamente maiores. Mesmo que os dados de FN apresentaram diferença estatística, os valores encontrados nas amostras de farinha foram considerados de ótima qualidade, pois se encontrou entre 200 a 350s (Perten Instruments, 2020).

Para o teor de glúten foi possível observar que as médias de PR01 $(27,69 \pm 1,50)$, PR02 $(27,62 \pm 1,80)$, PR04 $(28,10 \pm 1,73)$ e $\operatorname{RS} 02(28,93 \pm 1,87)$ foram significativamente iguais entre si $(p>0,05)$, e foram as que apresentaram as menores médias. A média de PR03 $(29,08 \pm 2,29)$ se diferenciou significativamente $(\mathrm{p}<0,05)$ das amostras PR01 e PR02, por apresentar média maior e a amostra RS01 $(29,78 \pm 1,83)$ se diferiu significativamente $(\mathrm{p}<0,05)$ das amostras PR01, PR02 e PR04, pelo 
mesmo motivo. Na panificação, o glúten tem como função reter os gases produzidos durante o processo de fermentação, fazendo assim que o pão tenha um volume satisfatório. Segundo Gutkoski (2007), a principal característica que uma farinha destinada para panificação precisa ter é uma estrutura em forma de rede, para que a mesma retenha os gases que se formam durante a fermentação e, assim, haja o crescimento dos pães. Quando o glúten formado for considerado forte, ou pouco extensível, ele gera um pão denso e sem volume, já se for considerado fraco, o pão produzido apresenta muitos buracos, já que não tem resistência e a rede de glúten acaba se rompendo com os gases da fermentação (Cauvain e Young, 2009). Por isso, avaliar o teor de glúten se torna necessário.

No entanto, as diferenças observadas para os valores de FN e de glúten não puderam ser determinadas pela origem das amostras, corroborando com os resultados apresentados anteriormente, pela análise de agrupamentos (Figura 4).

Tabela 1. Média dos resultados das análises de Falling number (FN), teor de glúten e alveografia: tenacidade (P) extensibilidade (L), energia de deformação da massa (W) e relação $P / L$ da farinha de trigo provenientes de diferentes locais do Estado do Paraná (PR01, PR02, PR03 e PR04) e Rio Grande do Sul (RS01 e RS02), entre os anos de 2014 e 2018, totalizando 30 análises.

\begin{tabular}{ccccccc}
\hline Variáveis & PR01 & PR02 & PR03 & PR04 & RS01 & RS02 \\
FN & $285,53 \pm 21,56^{\mathrm{a}, \mathrm{b}, \mathrm{c}}$ & $289,4 \pm 27,31^{\mathrm{a}, \mathrm{b}}$ & $266,73 \pm 26,63^{\mathrm{c}}$ & $298,03 \pm 19,61^{\mathrm{b}}$ & $291,47 \pm 27,61^{\mathrm{b}}$ & $291,87 \pm 34,60^{\mathrm{b}}$ \\
Glúten & $27,69 \pm 1,50^{\mathrm{a}}$ & $27,62 \pm 1,80^{\mathrm{a}}$ & $29,08 \pm 2,29^{\mathrm{b}}$ & $28,10 \pm 1,73^{\mathrm{a}, \mathrm{b}}$ & $29,78 \pm 1,83^{\mathrm{b}, \mathrm{c}}$ & $28,93 \pm 1,87^{\mathrm{a}, \mathrm{b}, \mathrm{c}}$ \\
$\mathbf{W}$ & $287,57 \pm 54,61^{\mathrm{a}}$ & $284,77 \pm 43,62^{\mathrm{a}}$ & $295,77 \pm 39,15^{\mathrm{a}}$ & $315,03 \pm 35,61^{\mathrm{a}}$ & $290,30 \pm 54,71^{\mathrm{a}}$ & $281,07 \pm 56,60^{\mathrm{a}}$ \\
$\mathbf{P}$ & $93,67 \pm 9,45^{\mathrm{a}}$ & $92,50 \pm 10,64^{\mathrm{a}}$ & $96,13 \pm 11,38^{\mathrm{a}}$ & $100,67 \pm 11,47^{\mathrm{a}}$ & $95,67 \pm 11,65^{\mathrm{a}}$ & $95,83 \pm 15,53^{\mathrm{a}}$ \\
$\mathbf{L}$ & $111,5 \pm 16,60^{\mathrm{a}}$ & $110,73 \pm 17,66^{\mathrm{a}}$ & $109,83 \pm 19,61^{\mathrm{a}}$ & $111,07 \pm 23,41^{\mathrm{a}}$ & $114,60 \pm 12,29^{\mathrm{a}}$ & $101,60 \pm 19,21^{\mathrm{a}}$ \\
$\mathbf{P} / \mathbf{L}$ & $0,85 \pm 0,16^{\mathrm{a}}$ & $0,86 \pm 0,18^{\mathrm{a}}$ & $0,93 \pm 0,33^{\mathrm{a}}$ & $1,00 \pm 0,49^{\mathrm{a}}$ & $0,85 \pm 0,14^{\mathrm{a}}$ & $0,98 \pm 0,26^{\mathrm{a}}$
\end{tabular}

Fonte: Autores.

Optou-se por tratar os dados padronizados da farinha de trigo, também pela aplicação da Análise dos Componentes Principais (PCA) onde, por combinações lineares das variáveis originais, dá-se a redução dos dados (Beebe; Pell; Seasholtz, 1998). Soeiro et al (2010), obtiveram bons resultados fazendo a aplicação da PCA na investigação da qualidade de farinhas de trigo e milho, composta por uma matriz de 30 linhas (amostras) e 7 colunas (variáveis), onde ficou clara a diferença da composição entre elas e que o tipo de embalagem utilizada para o armazenamento das farinhas poderia influenciar na quantidade das vitaminas analisadas.

Como pode ser observado na Tabela 2, será necessário utilizar os três primeiros componentes principais, onde CP1, CP2 e CP3 representam 38,085\%, 30,721 e 25,736\% respectivamente, apresentando uma variância total de 94,542\%. Pode-se, portanto, considerar que o modelo da PCA foi eficiente, uma vez que englobou mais de 70\% da variância dos dados nos primeiros componentes (Vicini e Souza, 2005). Além disso, pelo critério Kaiser-Gutman, o modelo também foi considerado satisfatório, uma vez que os três primeiros componentes principais (CP1, CP2 e CP3) apresentaram autovalores maiores que 1, de 3,01302, 2,43041 e 2,03603, respectivamente (Tabela 2) (PATIL et al, 2008). 
Tabela 2. Matriz das variáveis Falling number (FN), teor de glúten e alveografia: tenacidade (P), extensibilidade (L), energia de deformação da massa (W) e relação P/L da farinha de trigo, provenientes de diferentes locais do Estado do Paraná (PR01, PR02, PR03 e PR04) e Rio Grande do Sul (RS01 e RS02), entre os anos de 2014 e 2018, totalizando 30 análises.

\begin{tabular}{ccc}
\hline PC & Autovalor & \%Variância \\
1 & 3,01302 & 38,085 \\
2 & 2,43041 & 30,721 \\
3 & 2,03603 & 25,736 \\
4 & 0,323911 & 4,0943 \\
6 & 0,10788 & 1,3636 \\
\hline
\end{tabular}

Fonte: Autores.

A necessidade de utilizar os três primeiros eixos principais nesta análise expõem que há uma grande variabilidade nos parâmetros avaliados, que podem ser atribuídos a alguns fatores como às variações climáticas nos diferentes anos analisados, por exemplo, em 2016 o cultivo sofreu influência da La Niña, que trouxe um clima com menos umidade e excesso de chuvas, favorecendo as condições para a triticultura na região sul do Brasil, pois resulta em menor incidência de doenças fúngicas e problemas com grãos no pós-colheita, resultado em grãos de ótima qualidade e maior rendimento ao triticultor, mesmo com a redução da área plantada neste mesmo ano (Antunes, 2016). Outros fatores que podem ser considerados são a vasta variedade de cultivares para o trigo pão e a interação que sofre ainda no campo, com as condições de solo de cada região produzida, ataque de pragas, manejo, modo de colheita, armazenagem e moagem, onde pode resultar em diferentes valores para a quantidade e qualidade (força) do glúten e número de queda (Macritchie 2016). No entanto, todas as farinhas avaliadas no presente trabalho foram moídas e armazenadas sob as mesmas condições, logo, estas variações não puderam ser explicadas por estes parâmetros, podendo ser explicadas, provavelmente, pelas variações climáticas, de cultivo e do tipo de solo.

Como pode ser observado na Tabela 3, as variáveis mais representativas do eixo 1 foram $\mathrm{W}$ e $\mathrm{P}$, no eixo 2 P/L e no eixo $3 \mathrm{FN}$ e L e o glúten não está representado em nenhum dos três primeiros $\mathrm{CP}$, logo, não foi uma variável representativa para esta análise. A alveografia é uma análise que avalia algumas variáveis que são importantes quando se trata de produtos panificáveis, a variável $\mathrm{P}$, demonstra a resistência da massa e pode ser correlacionada a quantidade de água absorvida pela mesma, o excesso de resistência ou de tenacidade, como também é chamada, podendo influenciar no desenvolvimento pleno da massa. Quando se adiciona água mais uma força mecânica na farinha, ela demonstra sua força (W) para realizar essa tarefa, que se associa a capacidade de absorção da água (P) e a extensibilidade (L), que juntas, definem produtos de panificação com bom volume e boa textura (Módenes; Silva; Trigueros, 2009). Já a variável L indica a capacidade de extensibilidade da massa sem que ela se rompa e está ligada com o volume no processo fermentativo e a relação entre elas, o P/L, precisa ter um equilíbrio, pois o produto final está ligado fortemente a esta relação de resistência com extensibilidade (Germani, 2008). 
Research, Society and Development, v. 10, n. 4, e20710414021, 2021

(CC BY 4.0) | ISSN 2525-3409 | DOI: http://dx.doi.org/10.33448/rsd-v10i4.14021

Tabela 3. Contribuição de cada variável aos três primeiros componentes principais.

\begin{tabular}{cccc}
\hline PC & Eixo 1 & Eixo 2 & Eixo 3 \\
FN & $-1,6681$ & 0,73631 & $\mathbf{0 , 8 3 5 2 7}$ \\
GLÚTEN & $-0,51109$ & $-0,54839$ & $-1,7935$ \\
W & $\mathbf{0 , 9 7 3 0 4}$ & $-0,37869$ & 0,80047 \\
P & $\mathbf{0 , 8 3 7 5 5}$ & 0,52114 & $-0,015561$ \\
L & $-0,16214$ & $-1,523$ & $\mathbf{0 , 5 5 0 1 4}$ \\
P/L & 0,53074 & $\mathbf{1 , 1 9 2 7}$ & -0.37682 \\
\hline
\end{tabular}

Fonte: Autores.

Com base nos resultados apresentados na Figura 5, pôde-se verificar uma relação entre a variável força (W), capacidade de absorção de água $(\mathrm{P})$ e a relação P/L, mas que se contrapõem as variáveis extensibilidade (L) e FN. No CP1 é onde se localizam as variáveis mais representativas (80\%), pode-se sugerir, então, que a relação de equilíbrio entre P/L foi uma das mais representativas, junto com $\mathrm{P}$ e W. Visto que essas variáveis (W, P, L e P/L) são resultados de uma simulação do comportamento da massa durante a fermentação, portanto, estas estão relacionadas entre si (Zardo, 2010).

O FN é utilizado para determinar a atividade enzimática da alfa-amilase, que está fortemente ligada a fatores como: a germinação do grão, ainda no campo, ou a síntese da enzima durante a maturação do grão já colhido (Xu et. al. 2007). 
Figura 5. Análise dos componentes principais - Biplot: (a) do Eixo 1 (Componente 1) e Eixo 2 (Componente 2); (b) do Eixo 1 (Componente 1) e Eixo 3 (Componente 3), das farinhas de trigo processadas com trigos oriundos de diferentes locais do Estado do Paraná (PR01, PR02, PR03 e PR04) e Rio Grande do Sul (RS01 e RS02), entre os anos de 2014 e 2018, totalizando 30 análises.

(a)

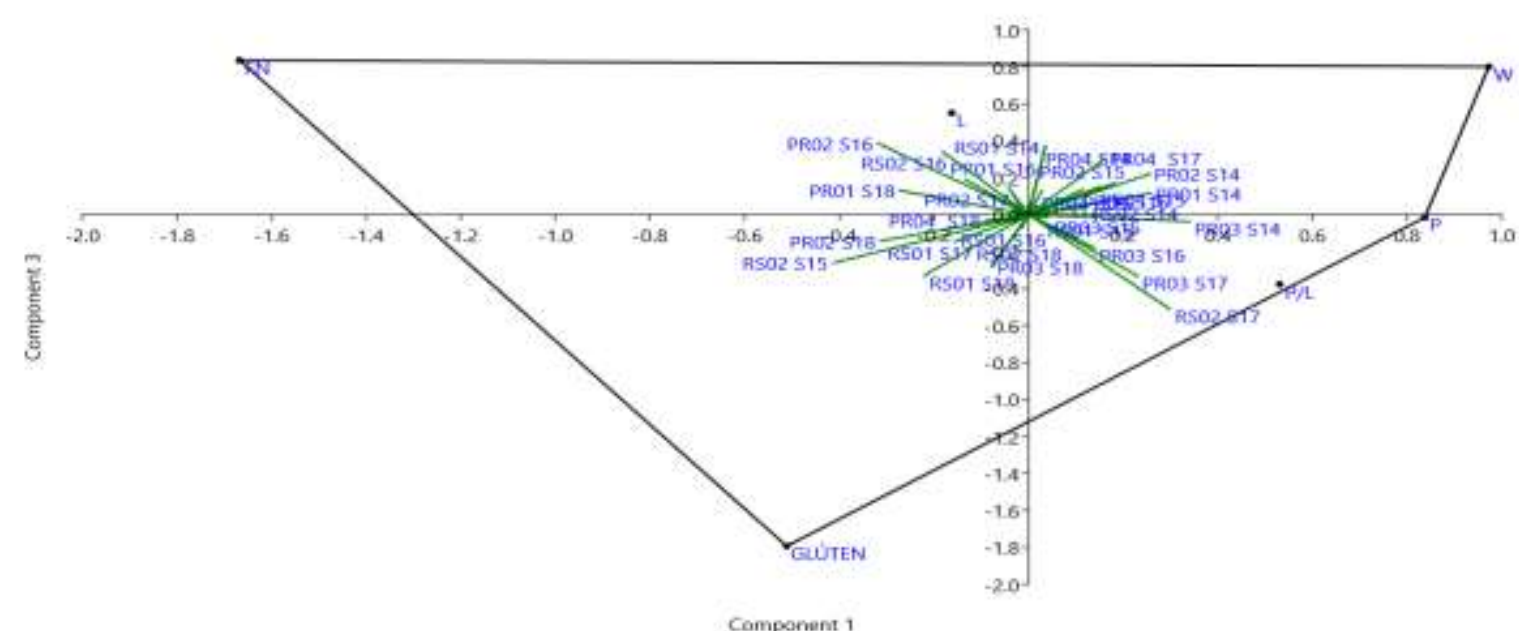

(b)

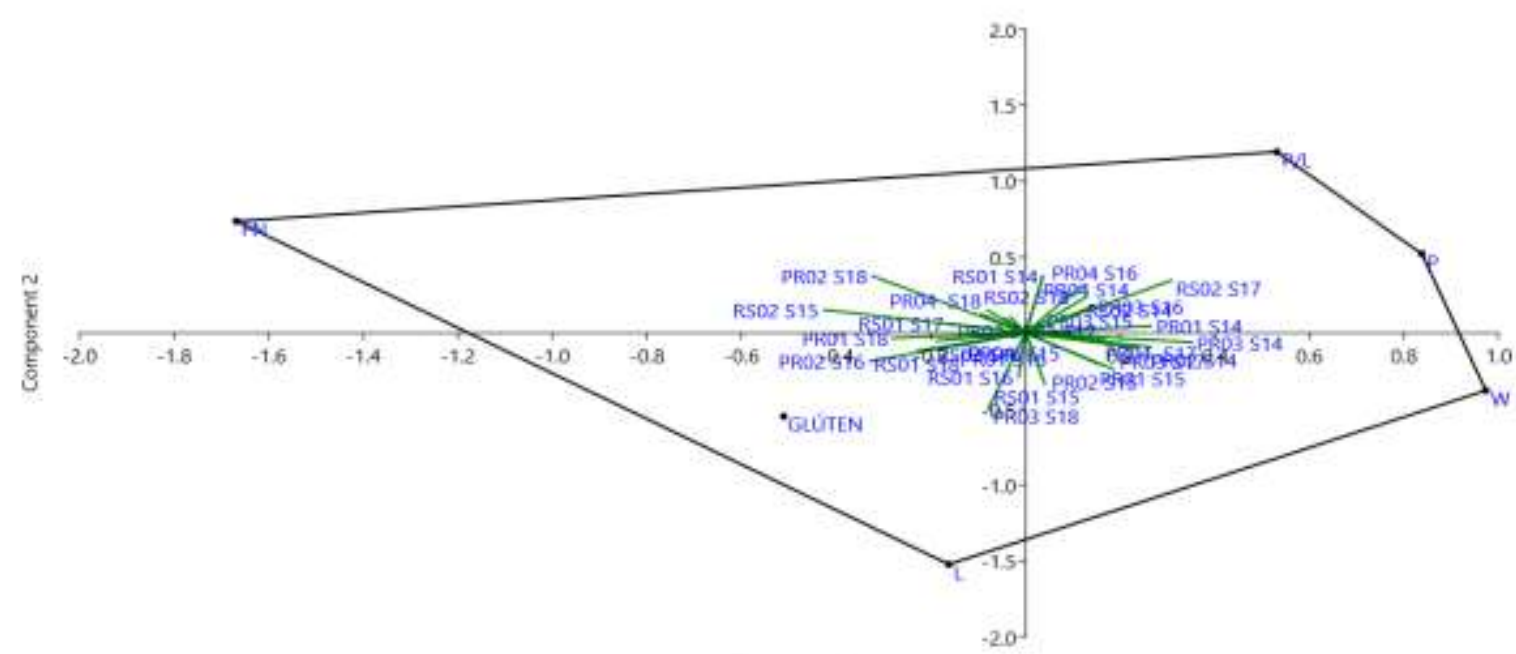

Component 1

Fonte: Autores.

Além disso, a atividade enzimática de uma farinha, tem importância para a indústria, visto que tem influência direta sobre o produto final a ser produzido, permitindo estimar a capacidade da fermentação da massa para a panificação. Quando a atividade enzimática se encontra entre 200 a 350 s o pão deve apresentar miolo firme, textura macia e grande volume, sendo valores ideais para a panificação. Já pães produzidos com uma farinha onde a atividade enzimática for alta (menor que $200 \mathrm{~s}$ ) ou baixa (maior que $350 \mathrm{~s}$ ) tendem a apresentar volume reduzido e características externas e internas não desejáveis (Perten Instruments, 2020). A matriz de distâncias, apresentada na Figura 4, já havia demonstrado que glúten e FN se distanciavam das demais. Na ACP o FN se localizou no CP3, mostrando que, das variáveis significativas, foi uma das que menos influenciou na qualidade das farinhas analisadas, juntamente com a variável extensibilidade (L). 
Cabe ressaltar que esse estudo é baseado em dados de trigos nacionais e que entidades e empresas, também brasileiras, estão sempre investindo e buscando melhorar a qualidade do grão nacional a cada safra. Quando o trigo nacional foi comparado com o importado encontrou-se diferenças, como foi o caso de Costa et. al. (2008), em que as variáveis de glúten e número de queda das amostras da farinha importada apresentaram maiores valores dos que as farinhas nacionais e que por meio da análise estatística apresentaram diferenças significativas $(p<0,05)$ entre si. No entanto, para a indústria moageira não existe farinha boa ou ruim e sim farinha adequada ou não para um determinado fim, ou seja, a indústria faz o uso da técnica de mescla de trigos ou de farinhas com diferentes teores de glúten e FN, ou o uso de enzimas, a fim de alcançar parâmetros desejáveis para a fabricação de biscoito, panificação ou massas.

Logo, o presente trabalho demonstrou que a qualidade da farinha de trigo analisada classificada como Trigo pão, todas produzidas no Brasil, apresentavam parâmetros de qualidade ideias, dentro dos parâmetros recomendados pela legislação vigente, demonstrando que a farinha de trigo brasileira apresenta potencial de competitividade no mercado. Logo, este trabalho contribuiu para encontrar onde estavam as divergências entre as variáveis que determinaram a qualidade da farinha de trigo, a fim de auxiliar a indústria na busca por respostas mais assertivas diante das dificuldades impostas, quando há necessidade de atender as especificações particulares do mercado.

\section{Considerações Finais}

Com relação aos resultados encontrados para o grão de trigo, para as variáveis de PH e umidade, todas as amostras encontravam-se dentro do que preconiza a legislação vigente. Não foi possível determinar uma tendência, uma vez que todas as variáveis analisadas apresentaram similaridade entre si, a respeito ao dendograma. $\mathrm{Na}$ a aplicação da PCA, nos resultados encontrados puderam-se observar que a variável glúten não foi explicada entre os três primeiros $\mathrm{CP}$, conforme demonstrado na Tabela 3, ficando estabelecido que as variáveis que precisam ser melhoradas, a fim de padronizar a farinha de trigo, destinada para a panificação, no local de estudo, foram as menos representativas, neste caso: FN (CP3) e glúten, mesmas variáveis que apresentaram resultados significativos para a análise de diferença de médias.

Com base nos resultados, este trabalho pode contribuir com a indústria, apresentando as variáveis que melhor colaboram para a qualidade da farinha destinada para panificação, e podem nortear a tomada de decisões mais rápidas e assertivas, evitando reprocessos na produção, ganhando tempo e volume produzido.

Sugere-se que trabalhos futuros como a comparação de trigos nacionais e internacionais nesta mesma metodologia, bem como o acompanhamento das modificações durante o armazenamento, para verificar possíveis alterações na qualidade tecnológica do trigo, desde que é recebido até sua utilização e testes de panificação para observação e verificação da qualidade tecnológica da farinha de trigo de diferentes locais e safras.

\section{Referências}

Aacc - American association of cereal chemists. (1999). Approved methods of the aacc. (8a ed.), Saint Paul.

Aacc - American association of cereal chemists. (2000). Approved methods of the aacc. (8a ed.), Saint Paul.

Abitrigo. (2020). Associação brasileira do trigo. Estatísticas. http://www.abitrigo.com.br/categoria-estatisticas/trigo/.

Abitrigo (2020). Associação brasileira do trigo. Raio X da cadeia do trigo e dos moinhos brasileiros. http://www.abitrigo.com.br/conhecimento/conhecimentoraio-X.

Amorim M, A. G.; Sousa, T. A. \& Souza, A. O. (2012) Determinação do pH e acidez titulável da farinha de semente de abóbora. Congresso norte e nordeste pesquisa e inovação. Tocantins.

Antunes, J. M. (2016). Clima favorável para o trigo na região sul. Notícia/12132979-Embrapa.

Araújo, H. M. C., Araújo, W. M. C., Botelho, R. B. A. \& Zandonadi, R. P. (2010). Doença celíaca, hábitos e práticas alimentares e qualidade de vida; Revista de Nutrição. Campinas, 23(3), 467-474, 
Battisti, R., Somavilla, L., Busanello, C. \& Schwerz, L. (2011). Eficiência do uso da massa hectolitro como teste rápido de vigor de semente de trigo (Triticum Aestivum). Revista da Fzva. Uruguaiana, 18(1), 125-135.

Beebe, K. R., Pell, R. J. \& Seasholtz, M. B. (1998). Chemometrics: a practical guide. John Wiley\&Sons.

Boschini, A. P. M. (2010). Produtividade e qualidade de grãos de trigo influenciados por nitrogênio e lâminas de água no Distrito Federal. Brasília. Faculdade de Agronomia e Medicina Veterinária, Universidade de Brasília.

Brasil, Mapa: Ministério da agricultura, pecuária e abastecimento. (2005). Instrução Normativa $N^{\circ}$ 8, de 03 de junho de 2005. Brasília, DF.

Brasil, Mapa: Ministério da agricultura, pecuária e abastecimento (2010). Instrução Normativa $N^{\circ} 38$, de 30 de novembro de 2010. Brasília, DF.

Bushuk, W. (1985). Flour proteins: structure and functionality in dough and bread. Cereal Foods World, 30(7), 447-451.

Caldeira, N. Q. N., Lima, Z. L. A., Seki, A. R. \& Runjanek, F. D. (2000) Diversidade de trigo, tipificação de farinhas e genotipagem. Revista Biotecnologia Ciência Desenvolvimento. Brasília.

Cauvain \& Young, S. P. (2009). Tecnologia da Panificação. (2a ed.), Editora Manole.

Costa, M. das G., Souza, E. L de., Stamford, T. L. M. \& Andrade, S. A. C. (2008). Qualidade tecnológica de grãos e farinhas de trigos nacionais e importados. Ciência e Tecnologia de Alimentos. Campinas. 28(1): 220-225.

Embrapa. (2009). Descrição dos métodos usados para avaliar a qualidade de trigo. Documentos online $\mathrm{N}^{\circ}$ 112. Embrapa Trigo. Passo Fundo. http://www.cnpt.embrapa.br/biblio/do/p_do112_5.htm.

Fano, A. (2015). Fontes de enxofre e manejo de nitrogênio na produtividade e qualidade industrial de trigo. UTFPR. Pato Branco. 69 f.: il.; CDD (22a ed.) 630.

Germani, R. (2008). Características dos grãos e farinhas de trigo e avaliação de suas qualidades. Embrapa - Laboratório de análise de trigo.

Guarienti, E. M. (1996). Qualidade industrial de trigo. Embrapa-Cntp.

Gutkoski, L. C., Klein, B., Pagnussatt, F. A. \& Pedó, I. (2007). Características tecnológicas de genótipos de trigo (Triticum aestivum L.) cultivados no cerrado. Ciência Agrotecnologia, 31, 786-792.

Hemery, Y. M., Fontan, L., Laillou, A., Jallier, V., Moench-Pfanner, R., Avallone, S. \& Berger, J. (2020). Influence of storage and packaging conditions of fortified wheat flour on microbial load and stability of folate and vitamin b12. Food Chemistry.

Jamrógiewicz, M. (2012). Application of the near-infrared spectroscopy in the pharmaceutical technology. Journal of Pharmaceutical and Biomedical Analysis, 66, 1-10.

Laranzini, D. P. (2015). Controle de qualidade aplicado a farinha de trigo panificável produzida em moinhos do estado do Paraná. Universidade Tecnológica Federal do Paraná - UTFPR. Francisco Beltrão.

Macritchie, F. (2016). Seventy years of research into breadmaking quality. Journal of Cereal Science, 70, 123-131.

Marathe, S. A., Machaiah, J. P., Rao, B. Y. K., Pednekar, M. D. \& Rao, V. S. (2002). Extension of shelf-life of whole-wheat flour by gamma radiation. International Journal of Food Science \& Technology. 37(2), 163-168.

Módenes, A. N.; Silva, A. M. da. |\& Trigueros, D. E. G. (2009). Avaliação das propriedades reológicas do trigo armazenado. Ciência e Tecnologia de Alimentos.

Patil, V. H., Singh, S. N., Mishra, S. \& Donavan, D. T. (2008). Efficient theory development and factor retention criteria: Abandon the 'eigenvalue greater than one' criterion. Journal of Business Research, 61(2), 162-170.

Perten instruments. (2020). Falling number. https://www.perten.com/Products/Falling-Number/.

Sabillon, L. \& Bianchini, A. (2016). From field to table: a review on the microbiological quality and safety of wheat-based products. Cereal Chemistry. V. 93, N.2, P 201 105-115.

Santos, C. M., Nalevaiko, F. K. S., Silva, P. M. L. \& Carvalho, P. A. (2015). Métodos analíticos aplicados pela granotec/granolab ao trigo e a farinha de trigo. $1^{\circ}$ Edição, Curitiba. Granolab Brasil.

Schmidt, D. A. M., Carvalho, F. I. F., Oliveira, A. C., Silva, J. A. G., Bertan, I., Valério, I. P., Hartwig, I., Silveira, G. \& Gutkoski, L. C. (2009). Variabilidade genética em trigos brasileiros a partir de caracteres componentes da qualidade industrial e produção de grãos. 68(1), 43-52.

Silva, R. C. da., Pino, L. M., Spoto, M. H. F. \& D’arce, M. A. B. R. (2010). Estabilidade oxidativa e sensorial de farinhas de trigo e fubá irradiados. Ciência Tecnologia de Alimentos, Campinas, 30(2): 406-413.

Soeiro, B. T., Boen, T. R., Filho, E. R. P. \& Pallone, J. A. L. (2010). Enriched flour quality investigation using Principal Component Analysis (PCA). Food Science and Technology.

Vicini, L. \& Souza, A.M. (2005). Análise multivariada: da teoria à prática. UFSM.

Xu, J., Bietz, J. A. \& Carriere, C. V. (2007). Viscoelastic properties of wheat gliadin and glutein suspension. Food Chemistry. Reading, 101(3), 1025-1030. 
Research, Society and Development, v. 10, n. 4, e20710414021, 2021

(CC BY 4.0) | ISSN 2525-3409 | DOI: http://dx.doi.org/10.33448/rsd-v10i4.14021

Zardo, F. P. (2010). Análises Laboratoriais para o controle de qualidade da farinha de trigo. Trabalho de Conclusão de Curso- Instituto Federal de Ciência e Tecnologia do Rio Grande do Sul, Bento Gonçalves. 